\title{
Stratified analysis reveals chemokine-like factor (CKLF) as a potential prognostic marker in the MSI-immune consensus molecular subtype CMS1 of colorectal cancer
}

\author{
Philip D. Dunne ${ }^{1}$, Paul G. O'Reilly ${ }^{1}$, Helen G. Coleman' ${ }^{2}$, Ronan T. Gray ${ }^{2}$, Daniel B. \\ Longley ${ }^{1}$, Patrick G. Johnston ${ }^{1}$, Manuel Salto-Tellez ${ }^{1}$, Mark Lawler ${ }^{1, *}$, Darragh G. \\ McArt ${ }^{1, *}$ \\ ${ }^{1}$ Centre for Cancer Research and Cell Biology, Faculty of Medicine, Health and Life Sciences, Queen's University Belfast, \\ Belfast, UK \\ ${ }^{2}$ Centre for Public Health, Faculty of Medicine, Health and Life Sciences, Queen's University Belfast, Belfast, UK \\ *These authors have contributed equally to this work \\ Correspondence to: Mark Lawler, e-mail: mark.lawler@qub.ac.uk \\ Keywords: colorectal cancer, gene expression profiling, molecular stratification, relapse risk, chemokine-like factor \\ Received: February 15, $2016 \quad$ Accepted: April 16, $2016 \quad$ Published: May 2, 2016
}

\section{ABSTRACT}

The Colorectal Cancer (CRC) Subtyping Consortium (CRCSC) recently published four consensus molecular subtypes (CMS's) representing the underlying biology in CRC. The Microsatellite Instable (MSI) immune group, CMS1, has a favorable prognosis in early stage disease, but paradoxically has the worst prognosis following relapse, suggesting the presence of factors enabling neoplastic cells to circumvent this immune response. To identify the genes influencing subsequent poor prognosis in CMS1, we analyzed this subtype, centered on risk of relapse.

In a cohort of early stage colon cancer $(n=460)$, we examined, in silico, changes in gene expression within the CMS1 subtype and demonstrated for the first time the favorable prognostic value of chemokine-like factor (CKLF) gene expression in the adjuvant disease setting [HR=0.18, $\mathrm{CI}=0.04-0.89]$. In addition, using transcription profiles originating from cell sorted CRC tumors, we delineated the source of CKLF transcription within the colorectal tumor microenvironment to the leukocyte component of these tumors. Further to this, we confirmed that CKLF gene expression is confined to distinct immune subsets in whole blood samples and primary cell lines, highlighting CKLF as a potential immune cell-derived factor promoting tumor immunesurveillance of nascent neoplastic cells, particularly in CMS1 tumors. Building on the recently reported CRCSC data, we provide compelling evidence that leukocyteinfiltrate derived CKLF expression is a candidate biomarker of favorable prognosis, specifically in MSI-immune stage II/III disease.

\section{INTRODUCTION}

Until recently, the classification of $\mathrm{CRC}$ has been limited to the generation of prognostic signatures based on gene expression profiles developed by supervised analysis for prognosis. Between 2012 and 2014, a number of studies underpinned a comprehensive molecular characterization of CRC [3-7], culminating in the landmark publication of consensus molecular subtypes by the Colorectal Cancer Subtyping Consortium (CRCSC) [8]. The four Consensus Molecular Subtypes (CMS) identified were: CMS1: microsatellite instability (MSI) immune (frequency 14\%); CMS2: canonical (37\%); CMS3: metabolic (13\%) and CMS4: mesenchymal (23\%), providing a more granular discrimination of the underlying CRC disease biology and permitting for the first time an attempt at dissecting the clinical utility of these robust subtypes. The CMS1 subtype is characterized by a high mutation burden, due to the abundance of MSI tumors within this subtype, and a high level of immune infiltration. This subgroup has long been associated with a relatively good prognosis in early stage disease. Paradoxically however, while 
only a relatively small proportion of patients with MSI tumors subsequently relapse, they have the worst overall prognosis [8-11].

Tumor-associated leukocytes (TALs) have previously been associated with favorable prognosis in CRC, particularly when the density and distribution of $\mathrm{CD} 8+$ and CD45RO + memory $\mathrm{T}$ cells are assessed [9, 12]. Recently, these findings were investigated using a pan-cancer meta-analytical framework combining gene expression profiles with survival data to assign prognostic value to gene transcripts [13]. Across a variety of cancers, this approach demonstrated an association between an increased abundance of immune-response genes and improved survival, confirming the generally favorable prognostic value of immune infiltration. While the presence of high immune infiltration is generally associated with improved cancer survival rates, paradoxically the presence of certain subpopulations of $\mathrm{T}$ cells can also be associated with poor prognosis [14]. The tissue-specific prognostic value of immune cell infiltration was further highlighted when the association between 22 subsets of TALs and patient survival was examined across 25 cancer types [13]. This pan-cancer approach further revealed the reciprocal, and sometimes counter-intuitive, nature of TAL prognostic associations based on cancer type. But while these published studies suggest that particular immune cell subsets are associated with prognosis in specific cancers; they do not take into account the presence of the emerging stratified subtypes within each cancer type.

To address this question, we employed a stratified approach, specifically in the immune-rich CMS1, to examine the prognostic value of individual gene transcription probesets by supervised risk analysis. This approach highlighted the probesets associated with relapse in the adjuvant stage II/III disease setting. Further survival analysis revealed the prognostic value of our findings specifically in the CMS1, thus validating this stratified analysis approach. Finally, we used gene expression profiles to delineate the source of expression of the identified gene across a range of specific human tissues and primary cells.

\section{RESULTS}

\section{Stratified approach to generate subgroup specific relapse rates}

We analyzed available data from the stage II/III colon cancer tumors in the reference dataset employed by the CRCSC (GSE39582) [7]. Within the overall cohort of $n=460$ Stage II and III colon cancer cases (Figure 1), $56 \%$ were male, $56 \%$ were Stage II, $41 \%$ were proximal colon tumors, $44 \%$ received adjuvant treatment and the mean (SD) age at diagnosis was 68 (13) years (Table 1). Individuals with CMS1 tumors were significantly more likely to have proximal colon tumors ( 78 v $33 \%, \mathrm{p}<0.001$ ), less likely to receive adjuvant treatment (25 v 48\%, $\mathrm{p}<0.001$ ) and were older (mean $70 \vee 67$ years, $\mathrm{p}=0.05$ ) compared with individuals with other tumor classifications within the cohort (Table 1). No differences in stage or sex distribution were detected between individuals with CMS1 and other tumor classifications.

We then examined the prognostic value of the four identified subtypes (CMS1-4) based on 3-year relapse risk using a cohort of $n=177$ untreated stage II/III patient profiles with CMS assignment and complete clinical follow up data (Figure 1). As previously described, there was a trend towards better prognosis in the MSI-immune CMS1 compared to the CMS4 mesenchymal subgroup (3year relapse rate $(\mathrm{RR})$ of $13 \%$ vs. $31 \%, \mathrm{HR}=0.40,95 \% \mathrm{CI}$ $0.15-1.03$ ) (Figure 2 and Supplementary Table 1).

\section{Gene expression associated with risk in the CMS1}

Using clinical relapse data, we generated an initial discovery subset (detailed in Materials and Methods) resulting in 46 stringently filtered transcriptional profiles specifically assigned to CMS1 (Figure 1). As the frequency of patients assigned to CMS1 in the CRCSC overall cohort was $14 \%$, this collection of 46 patients represents the largest possible publically available CMS1 microarray based dataset from a single cohort. Using an ANOVA model of high risk contrasted with low risk to examine true prognostic biology within CMS1, we generated a list of differentially expressed probesets. This resulted in 55 annotated probesets accounting for 49 unique genes (Table 2). While the majority of these genes were represented only once, Chemokine-Like Factor (CKLF) was represented by all three probesets corresponding to this gene on the microarray platform, highlighting its association with relapse risk.

\section{Clinical relevance of CKLF gene expression in the CMS1}

To validate the clinical value of our findings, we examined the stage II/III profiles with the complete data in GSE39582 (Figure 1) [7] by removing all the imposed restraints from the initial discovery subset to create a survival validation subset. Using mean expression of the three probesets representing CKLF, we stratified tumor profiles across all CMSs into high, medium and low, based on CKLF gene expression. When comparing the highest with the lowest levels of CKLF expression, no significant associations with recurrence-free survival were detected in the overall cohort (Table 3 and Supplementary Figure 1A and $1 \mathrm{~B})$ with each group overlapping.

However, when the CMS1 patient profiles were stratified by CKLF expression separately (CMS1 profiles in the survival validation cohort $n=79$, Figure 

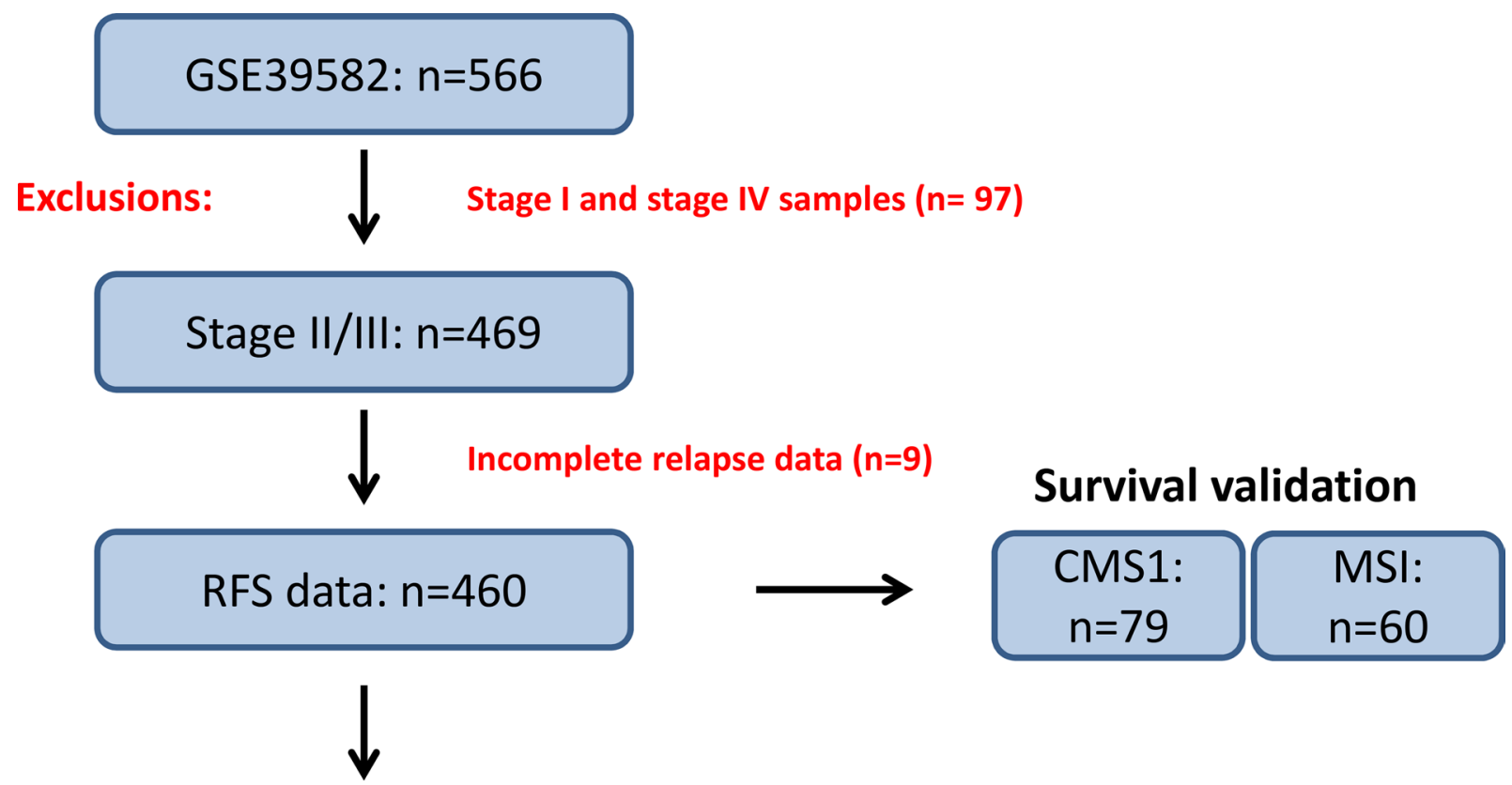

\section{Discovery subset}

Follow up less than 36 months or
relapse after 36 months $(n=88)$

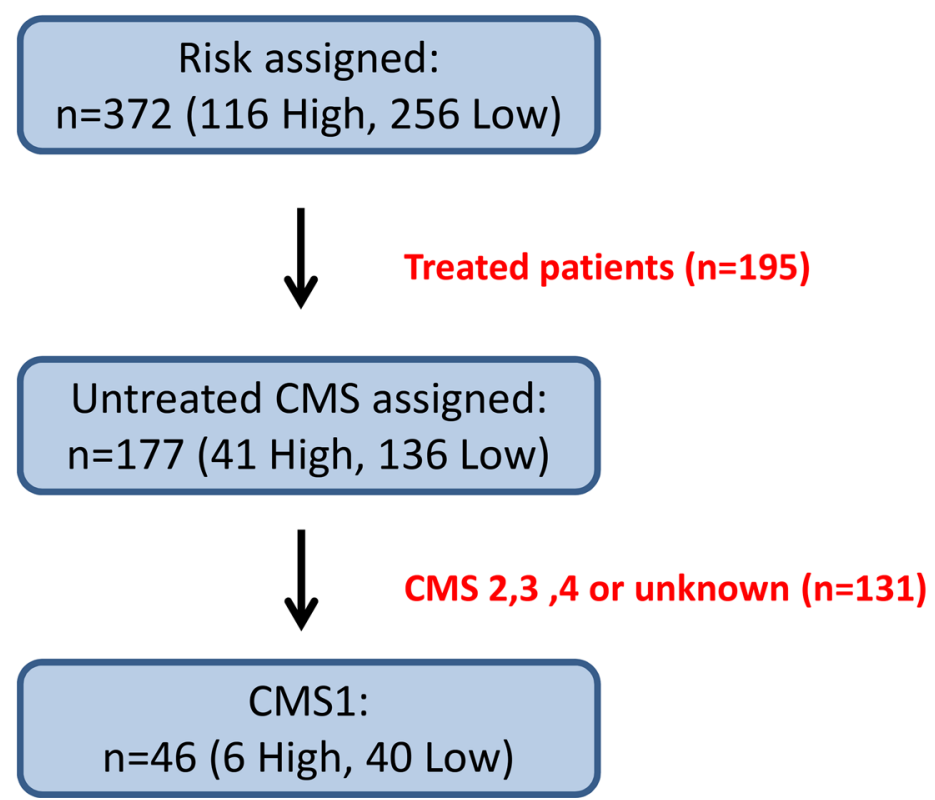

Figure 1: Study overview of discovery and survival validation subsets. The data used in this study was obtained from 566 Affymetrix U133 Plus 2.0 patient transcriptional profiles accessed through the NCBI GEO accession number GSE39582. Filtering for stage II/III and complete relapse data reduced this cohort to 460 profiles. The CMS1 specific discovery subset was composed of 46 tumors which fulfilled risk filtering (see Materials and Methods) followed by differential gene expression analysis based on risk classification. The survival validation subset was composed of either the entire cohort, CMS1 specific or MSI specific subgroups of samples. For relapse-free survival analyses, only stage II and III patients were considered, giving 79 transcriptional profiles in the CMS1 or 60 transcriptional profiles in the MSI subgroups. 
Table 1: Characteristics of patients in CMS1 (Consensus Molecular Subtype) compared with other tumor subtypes

\begin{tabular}{|c|c|c|c|}
\hline Characteristic & $\begin{array}{c}\text { CMS } 1 \\
n=80\end{array}$ & $\begin{array}{l}\text { All other subtypes* } \\
\qquad \mathbf{n}=\mathbf{3 8 0}\end{array}$ & p-value \\
\hline Age, years, mean (SD) & $70.2(14.6)$ & $67.0(12.0)$ & 0.05 \\
\hline $\begin{array}{l}\text { Sex, n }(\%) \\
\text { Male } \\
\text { Females }\end{array}$ & $\begin{array}{l}38(47.5) \\
42(52.5)\end{array}$ & $\begin{array}{l}220(57.9) \\
160(42.1)\end{array}$ & 0.09 \\
\hline $\begin{array}{l}\text { Tumour stage, n (\%) } \\
\text { II } \\
\text { III }\end{array}$ & $\begin{array}{l}48(60.0) \\
32(40.0)\end{array}$ & $\begin{array}{l}211(55.5) \\
169(44.5)\end{array}$ & 0.46 \\
\hline $\begin{array}{l}\text { Tumour location, } \mathbf{n}(\%) \\
\text { Proximal } \\
\text { Distal }\end{array}$ & $\begin{array}{l}62(77.5) \\
18(22.5)\end{array}$ & $\begin{array}{l}125(32.9) \\
255(67.1)\end{array}$ & $<0.001$ \\
\hline $\begin{array}{l}\text { Adjuvant treatment receipt, n (\%) } \\
\text { Yes }\end{array}$ & $20(25.0)$ & $182(47.9)$ & $<0.001$ \\
\hline
\end{tabular}

Comparative analysis of age, sex, stage, location and treatment for CMS1 $(n=80)$ patients versus the remaining CMS 2, 3, 4 and unclassified patients $(\mathrm{n}=380)$.

*Comprises n=193 CMS2, n=50 CMS3, n=99 CMS4 and n=38 unclassified CMS subtypes.

\section{CMS prognosis}

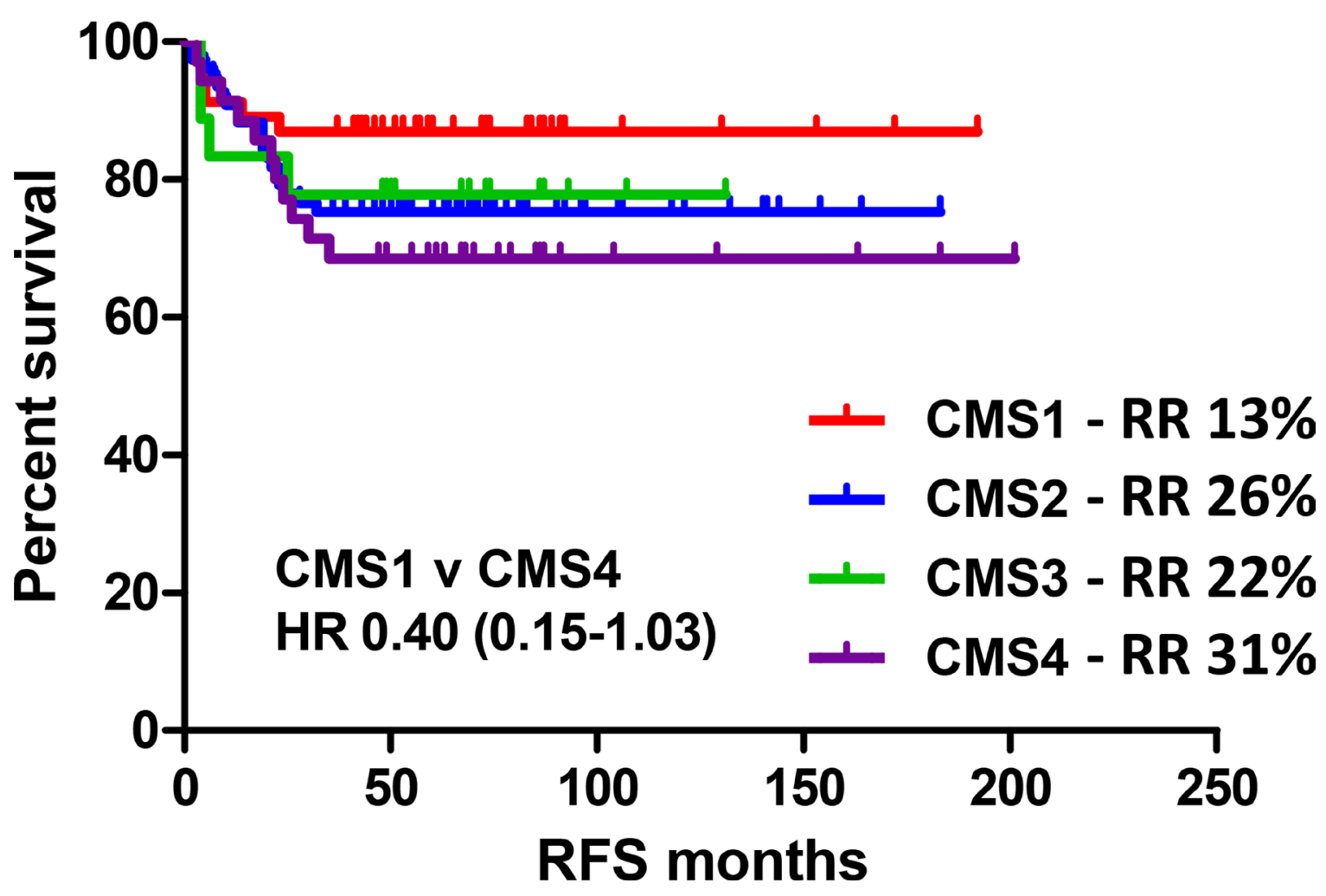

Figure 2: Relapse risk in recently defined consensus molecular subtypes. Survival curve using Kaplan-Meier estimation comparing prognosis of CMS1-4 in untreated stage II/III CRC patients (GSE39582). RR indicates the 3 year relapse rate. 
Table 2: Probesets and genes associated with relapse risk in CMS1

\begin{tabular}{|c|c|c|c|}
\hline Column ID & Gene Symbol & $\begin{array}{l}\text { p-value } \\
\text { (Risk) }\end{array}$ & $\begin{array}{c}\text { Fold-Change } \\
\text { (High Risk vs. Low Risk) }\end{array}$ \\
\hline 221058_s_at & CKLF & 0.000302824 & -1.56349 \\
\hline 242465_at & LOC 100505592 & 0.000332139 & -1.78683 \\
\hline 223451_s_at & CKLF & 0.000598022 & -1.62852 \\
\hline 219161_s_at & CKLF & 0.0019177 & -1.56255 \\
\hline 209759_s_at & DCI & 0.00192191 & -1.51586 \\
\hline 213285_at & TMEM30B & 0.00219006 & -1.86942 \\
\hline 229331_at & SPATA18 & 0.0022411 & -2.15593 \\
\hline 210002_at & GATA6 & 0.00239525 & -1.95547 \\
\hline 203814_s_at & NQO2 & 0.00317772 & -1.88969 \\
\hline 232809_s_at & FLT1 & 0.00388947 & -1.58036 \\
\hline 221958_s_at & WLS & 0.00428383 & -1.90076 \\
\hline 228360_at & LYPD6B & 0.00460096 & -2.18477 \\
\hline Column ID & Gene Symbol & $\begin{array}{l}\text { p-value } \\
\text { (Risk) }\end{array}$ & $\begin{array}{c}\text { Fold-Change } \\
\text { (High Risk vs. Low Risk) }\end{array}$ \\
\hline 205728_at & ODZ1 & $7.48 \mathrm{E}-07$ & 1.63545 \\
\hline 208148_at & MYH4 & $1.31 \mathrm{E}-06$ & 2.36166 \\
\hline 214603_at & MAGEA2 /// MAGEA2B & $4.30 \mathrm{E}-05$ & 3.64337 \\
\hline 214642_x_at & MAGEA5 & $9.20 \mathrm{E}-05$ & 3.02798 \\
\hline 235700_at & CT45A1 & 0.000129371 & 2.11826 \\
\hline 219011_at & PLEKHA4 & 0.000144152 & 1.67177 \\
\hline 242577_at & LOC389834 & 0.000198257 & 1.57463 \\
\hline 1553830_s_at & MAGEA2 /// MAGEA2B & 0.000294457 & 2.9941 \\
\hline 210467_x_at & MAGEA12 & 0.00031194 & 3.36053 \\
\hline 220445_s_at & CSAG2 /// CSAG3 & 0.000344059 & 2.01273 \\
\hline 204086_at & PRAME & 0.000353129 & 2.95165 \\
\hline 233092_s_at & LOC 100271840 & 0.00043833 & 1.78984 \\
\hline 214612_x_at & MAGEA6 & 0.000469934 & 8.44036 \\
\hline 209942_x_at & MAGEA3 & 0.000595555 & 8.19105 \\
\hline 205563_at & KISS1 & 0.000821326 & 1.88576 \\
\hline 209733_at & MID2 & 0.000932513 & 1.72349 \\
\hline 215729_s_at & VGLL1 & 0.00106856 & 1.89873 \\
\hline 214254_at & MAGEA4 & 0.00109841 & 2.16359 \\
\hline 244631_at & LOC389834 & 0.00112161 & 1.97882 \\
\hline 1570445_a_at & LOC643201 & 0.00116794 & 1.89823 \\
\hline 232195_at & GPR158 & 0.00120259 & 2.43469 \\
\hline
\end{tabular}

(Continued) 


\begin{tabular}{|c|c|c|c|}
\hline Column ID & Gene Symbol & $\begin{array}{l}\text { p-value } \\
\text { (Risk) }\end{array}$ & $\begin{array}{c}\text { Fold-Change } \\
\text { (High Risk vs. Low Risk) }\end{array}$ \\
\hline 243683_at & MORF4L2 & 0.00163486 & 1.81374 \\
\hline 204823_at & NAV3 & 0.00181836 & 1.73127 \\
\hline 235004_at & RBM24 & 0.00183565 & 1.8934 \\
\hline 37028_at & PPP1R15A & 0.001906 & 1.72498 \\
\hline 210751_s_at & RGN & 0.00195509 & 1.52471 \\
\hline 202014_at & PPP1R15A & 0.00198168 & 1.73184 \\
\hline 229511_at & SMARCE1 & 0.00202072 & 1.70354 \\
\hline 210605_s_at & MFGE8 & 0.00205293 & 1.7212 \\
\hline 224825_at & DNTTIP1 & 0.00206935 & 1.64464 \\
\hline 227084_at & DTNA & 0.00217336 & 1.69962 \\
\hline 236840_at & C12orf56 & 0.00232896 & 1.98568 \\
\hline 220106_at & NPC1L1 & 0.00234885 & 1.71598 \\
\hline 1567912_s_at & CT45A1 & 0.00295615 & 1.75065 \\
\hline 227239_at & FAM126A & 0.00346844 & 2.32878 \\
\hline 208075_s_at & CCL7 & 0.00348212 & 1.85285 \\
\hline 236514_at & ACOT8 & 0.0037126 & 1.6944 \\
\hline 223138_s_at & DHX36 & 0.0038878 & 1.60102 \\
\hline 213906_at & MYBL1 & 0.00395218 & 2.28576 \\
\hline 235561_at & TXNL1 & 0.00418508 & 1.77398 \\
\hline 229252_at & ATG9B & 0.00418806 & 1.71331 \\
\hline 218853_s_at & MOSPD1 & 0.00482949 & 1.77617 \\
\hline 201023 at & TAF7 & 0.00499489 & 1.56954 \\
\hline
\end{tabular}

Differentially expressed probesets and their associated gene name between high and low risk groups in CMS1. Following ANOVA, fold-change $+/-1.5$ fold and $p$-value $<0.005$ filters were applied resulting in 55 annotated probesets associated with risk in CMS1. Positive fold-change indicates higher expression in high-risk group.

1) a $79 \%$ reduced risk of recurrence was evident for the highest compared with the lowest level of CKLF expression (Figure 3A, Table 3 and Supplementary Figure 1C and 1D). This risk of recurrence became statistically significant once confounding factors such as age, stage, tumor location and adjuvant treatment were adjusted for (HR $0.18,95 \%$ CI 0.04-0.89) (Table 3). The CKLF-medium group appeared to have an intermediate survival compared to the other two groups (Figure 3A), indicating that the positive prognostic value of CKLF was restricted to tumors within the highest-expressing tertile. These findings were also demonstrated using Euclidean and Ward clustering with a skewing of highrisk samples towards the low CKLF expression clusters (Supplementary Figure 1E). Using cross tabulation of the patient profiles, we found comparable numbers of BRAF mutants and wildtypes in both the high$(15 \%)$ and low-risk (17\%) groups, which ruled out the possibility of an inadvertent enrichment for BRAF mutants in the CMS1 high-risk subtype (Supplementary Table 2). In addition, we found no difference in CKLF gene expression across the entire cohort according to CMS (Supplementary Figure 1F), indicating the ubiquitous nature of CKLF gene expression in all CMSs. This trend towards a favorable prognosis in the CMS1 specific CKLF-high group was also observed in a further independent dataset (HR 0.38, 95\% CI 0.131.13) (GSE14333 $n=188$, CMS1 $n=41)$, containing a mix of colon and rectal tumor transcriptional profiles, some of which had received adjuvant chemotherapy or neo-adjuvant chemo-radiotherapy treatments regimes (Supplementary Figure 1G). 


\begin{tabular}{|c|c|c|c|c|}
\hline & \multicolumn{2}{|c|}{ CKLF gene expression* } & \multirow{2}{*}{$\begin{array}{c}\text { Unadjusted Hazard } \\
\text { ratios } \\
\begin{array}{c}\text { (95\% confidence } \\
\text { intervals) }\end{array} \\
\begin{array}{c}\text { High v. Low } \\
\text { expression }\end{array}\end{array}$} & \multirow{2}{*}{$\begin{array}{c}\text { Adjusted** Hazard } \\
\text { ratios } \\
(95 \% \text { confidence } \\
\text { intervals })\end{array}$} \\
\hline & $\begin{array}{c}\text { Number non-events } \\
\text { Low/Med/High }\end{array}$ & $\begin{array}{l}\text { Number events } \\
\text { Low/Med/High }\end{array}$ & & \\
\hline \multicolumn{5}{|l|}{ CMS1 } \\
\hline Untreated $(n=59)$ & $17 / 15 / 19$ & $5 / 2 / 1$ & $0.21(0.04-1.03)$ & $0.19(0.04-0.96)$ \\
\hline All $(n=79)$ & $20 / 19 / 25$ & $7 / 6 / 2$ & $0.21(0.04-1.03)$ & $0.18(0.04-0.89)$ \\
\hline \multicolumn{5}{|l|}{ MSI } \\
\hline Untreated $(n=47)$ & $12 / 13 / 16$ & $4 / 1 / 1$ & $0.18(0.02-1.65)$ & $0.19(0.02-1.85)$ \\
\hline $\operatorname{All}(n=60)$ & $15 / 16 / 19$ & $5 / 4 / 1$ & $0.17(0.01-1.42)$ & $0.16(0.02-1.39)$ \\
\hline \multicolumn{5}{|l|}{ Entire cohort } \\
\hline Untreated $(n=258)$ & $63 / 65 / 68$ & $20 / 19 / 23$ & $0.99(0.54-1.79)$ & $0.93(0.50-1.73)$ \\
\hline Treated $(n=202)$ & $43 / 41 / 41$ & $28 / 27 / 22$ & $0.80(0.45-1.43)$ & $0.90(0.50-1.62)$ \\
\hline All $(n=460)$ & $106 / 106 / 109$ & $48 / 46 / 45$ & $0.87(0.58-1.31)$ & $0.98(0.64-1.49)$ \\
\hline
\end{tabular}

RFS analysis was performed using Cox proportional hazards method in the CMS1, MSI or entire cohort stratified by CKLF expression levels. Analysis was performed both before and following adjustment.

*Cut-offs for low/medium/high CKLF gene expression based on tertile values.

**Adjusted for age, sex, TNM stage, tumour location and adjuvant treatment receipt (in treated analyses only).

\section{Prognostic value of CKLF gene expression in MSI patients}

Given that CMS1 is highly enriched for MSI tumors, we performed a further stratification, by selecting only MSI profiles from the stage II/III cohort, regardless of CMS clustering (Figure 1). As expected, these profiles were enriched for the CMS1 samples (49 CMS1 out of 60 MSI tumors $-82 \%)$. Patient tumors $(n=60)$ were stratified into three groups based on CKLF gene expression (Figure 3B). Analysis of the high expression compared to the low expression group in the MSI tumor profiles did reflect a similar trend, with an $84 \%$ reduced risk, but this result was not significant (HR 0.16, 95\% CI 0.02-1.39), indicating that CMS1 transcriptional classification is not simply a marker of MSI status (Table 3, Figure 3B).

\section{CKLF gene expression is associated with the tumor immune infiltrate}

Gene set enrichment analysis of the CMS1 group in the CRCSC study revealed an increased expression of genes associated with immune infiltration [8], confirming previous pathology-based associations between MSI and immune infiltrate $[10,11]$. Given the distinct structure of the tumor microenvironment (TME) within the CMS1 tumors, we sought to determine if the precise origin of
CKLF gene expression was confined to any specific cell type within the TME. To address this question, we utilized a separate microarray dataset obtained from dissociated fresh primary tumors (GSE39396), which had been Fluorescence Activated Cell Sorting (FACS) selected into specific endothelial, epithelial, leukocyte and fibroblast populations [15]. Assessment of the expression profiles according to the cell of origin confirmed that CKLF is significantly associated with tumor infiltrating leukocytes $(p<0.005)$ when compared to the other cell types represented within the TME (Figure 4A).

\section{CKLF gene expression profile across diverse human tissue and primary cells}

While CKLF gene expression is significantly associated with the leukocyte population within CRC tumors, we wished to comprehensively profile its expression levels in other human tissues and cells. Using the recently assembled Genotype-Tissue Expression (GTEx) portal [16, 17] (http://gtexportal.org/home), we examined the gene expression of CKLF (as measured by RNA-seq analysis) across a diverse range of 53 tissues (version V6, January 2016) to investigate the pattern of CKLF gene expression. This analysis revealed high expression of CKLF in whole blood samples, compared to all other tissues represented within the portal (subset 
shown in Figure 4B). The wide range of expression values observed for CKLF in these whole blood samples, represented by a large spread and outliers, indicates that there is clear variation across patient samples which may be due to the heterogeneous populations represented within whole blood. This finding was confirmed using a further cohort [18] from a panel of 79 human tissues (GSE1133) (subset shown in Supplementary Figure 2).

To further delineate the specific cell type expressing CKLF, we utilized the BioGPS database [19], which captures gene expression profiles across 745 samples representing a diverse range of primary human cells [20] including multiple immune sub-populations detectable in whole blood. Within this comprehensive collection of human cell types, we find CKLF expression levels are highly concordant in all three probesets across the entire cohort of cell types (Supplementary Figure 3). High CKLF gene expression was consistently associated with a small number of immune cell types including hematopoietic stem cells, neutrophils and the anti-tumorigenic $\gamma \delta$ T cells (gamma-delta T cells) (Figure 4C).

\section{DISCUSSION}

The recent publication of four consensus molecular subtypes in CRC has provided a framework for clinically relevant stratified-based discovery to be applied in $\mathrm{CRC}$ for the first time [8]. Using the reference dataset employed by the CRCSC [7], we specifically selected the MSI-immune CMS1 transcriptional profiles for analysis. Selection of this subtype of tumors in isolation allowed us to identify subtle transcriptional changes associated with relapse risk which would have otherwise been missed across an unstratified patient cohort.

Although stratified medicine does provide a unique opportunity for analyzing specific subtypes, particularly as we move away from the traditional two arm studies to multi-arm, multistage clinical trials, [21] one of the difficulties which will inevitably be encountered with the stratified approach is the analysis of selected small patient cohorts, which are difficult for traditional statistical models to assess. Additionally, as we focus on the good prognostic CMS1 subgroup, the number of relapse events can be
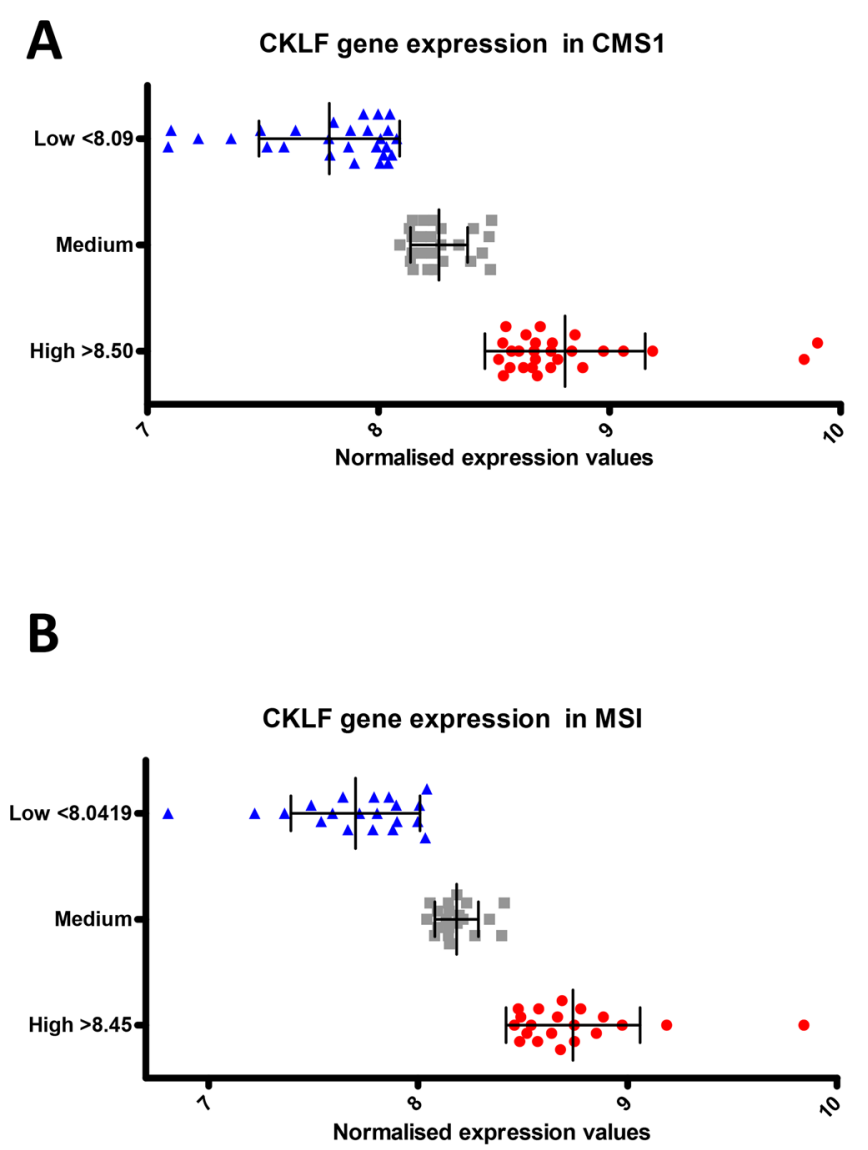

\section{CMS1}

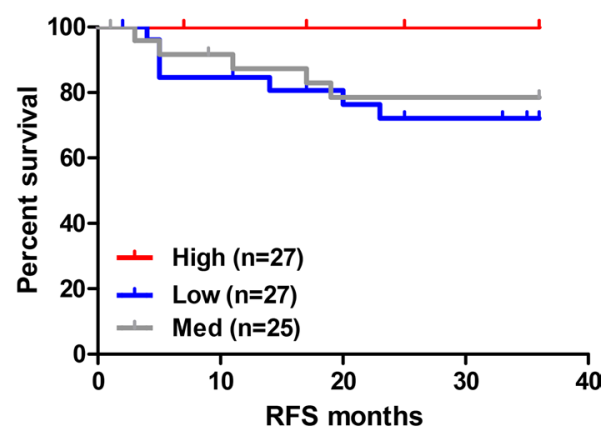

MSI

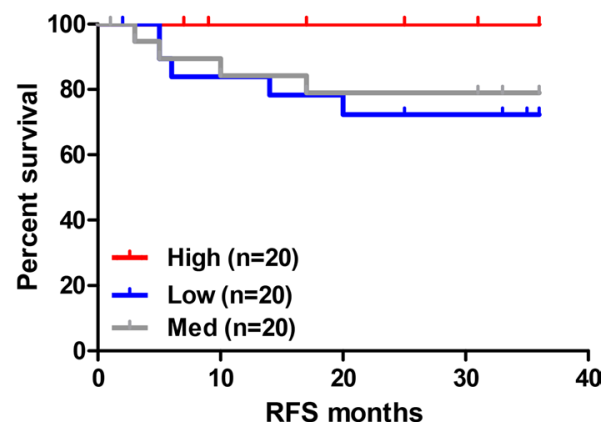

Figure 3: CKLF gene expression is associated with prognosis only in CMS1. A. Survival curve using Kaplan-Meier estimation comparing CKLF levels in CMS1 stage II/III CRC patients (GSE39582) B. Survival curve using Kaplan-Meier estimation comparing CKLF levels in MSI stage II/III CRC patients (GSE39582). 
A

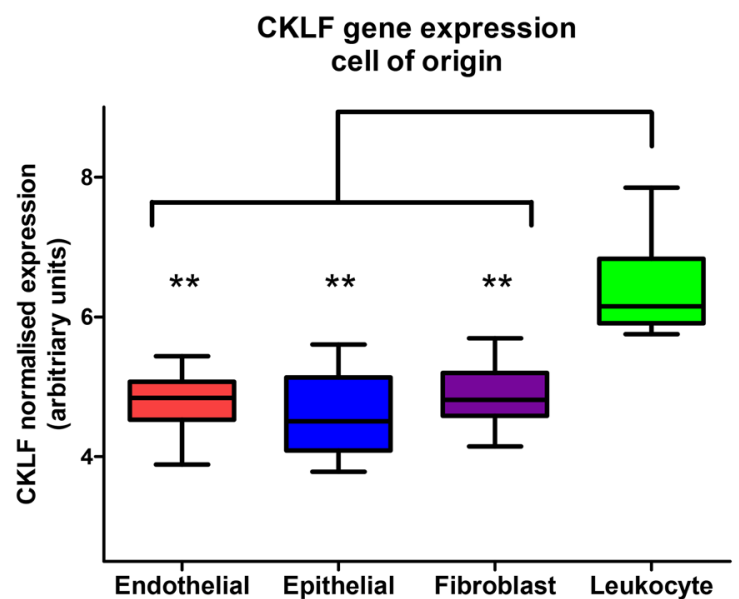

B

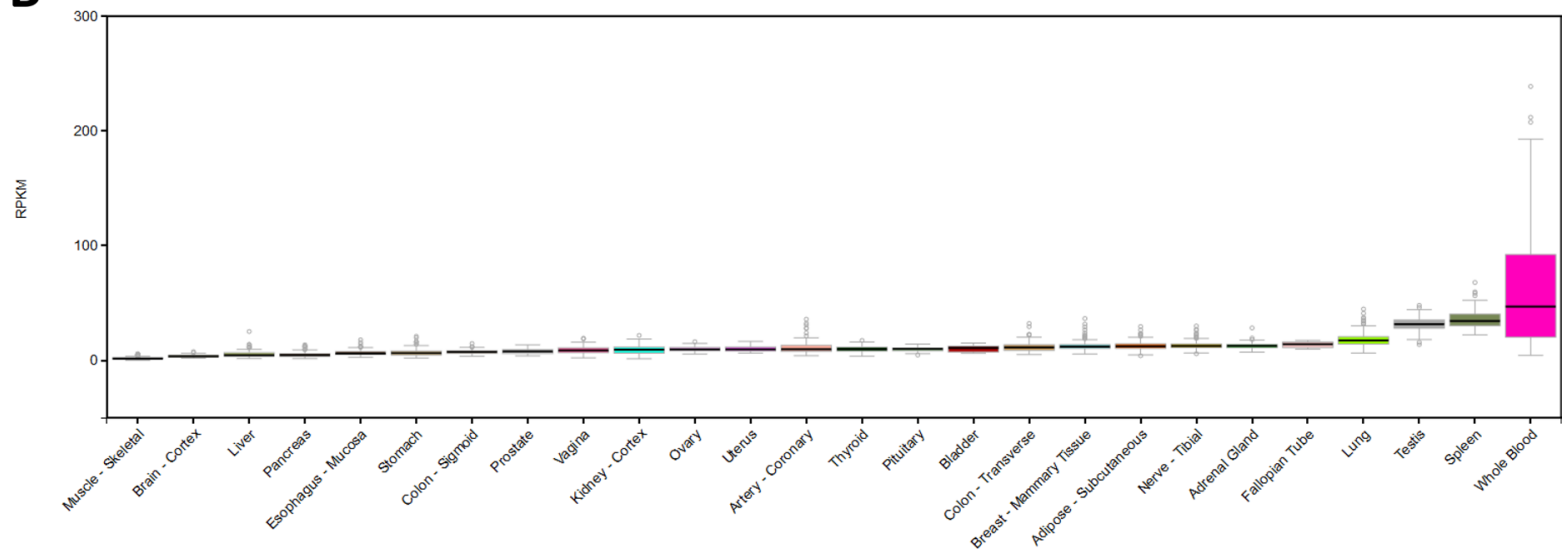

C

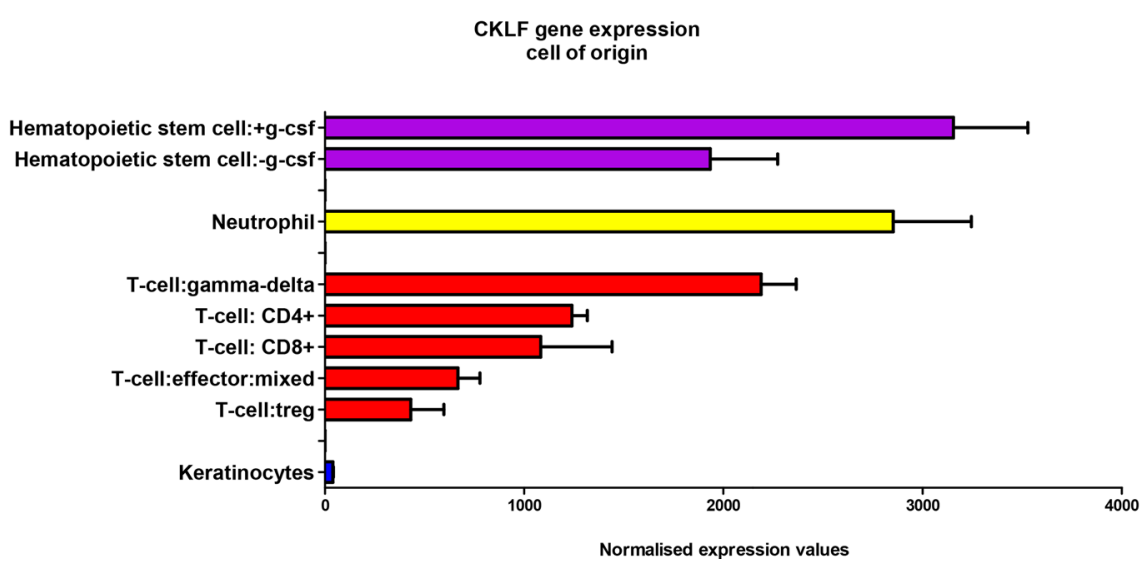

Figure 4: CKLF gene expression within the tumor microenvironment and normal tissue. A. Box and whisker plot of CKLF expression according to specific endothelial, epithelial, fibroblast or leukocyte cell-of-origin from FACS sorted primary colorectal tumors. B. CKLF RNA-seq expression values are shown in RPKM (Reads Per Kilobase of transcript per Million mapped reads), calculated from a gene model with isoforms collapsed to a single gene. No other normalization steps have been applied. Box plots are shown as median and 25th and 75th percentiles; points are displayed as outliers if they are above or below 1.5 times the interquartile range. The data used for the analyses described in this manuscript were obtained from the Genotype-Tissue Expression (GTEx) portal (http://gtexportal.org/home) version V6 in January 2016. C. CKLF gene expression from a subset of the Atlas of Human Primary Cells cohort. Bar charts are shown as median CKLF gene expression according to specific lineage and error bars represent standard deviation. $* *$ denotes $\mathrm{p}<0.005$. 
very low or even zero in some cases, which is problematic when performing initial proportional hazards fitness analysis. The precise application of stratified medicine will depend on how these statistical issues are resolved, but the model used in this study represents a pipeline approach of how an early discovery phase analysis can yield valuable information and generate hypotheses for validation in focused clinical studies.

This pipeline approach identified CKLF as a good prognostic marker in early stage CMS1 patients, where analysis of relapse rates highlighted the significant prognostic advantage for patients with high levels of CKLF gene expression. These findings indicate the potential for CKLF as a prognostic marker in this stratified group and provide a framework for further hypothesisdriven analysis in ascertaining its true clinical value. Our analysis also ruled out enrichment for BRAF mutations in the high-risk subgroup, as there are similar proportions of BRAF mutations represented in the low-risk group. While a similar prognostic trend for CKLF is also found in MSI tumor profiles, this data fails to reach significance, but may be limited by reduced statistical power in this analysis. It is the limitation of small patient numbers within stratified groups, which may be challenging when examining retrospective unselected cohorts using a stratified approach. This point is further highlighted given the size of the cohort we begin with, containing 566 patient transcriptional profiles, which represents one of the largest publically available single clinical CRC cohort datasets.

The CKLF protein was originally identified from a screen using a monocyte cell line and was found to have a strong chemotactic effect on human neutrophils, lymphocytes and monocytes [22]. CKLF was subsequently found to bind with the chemokine receptor CCR4, while increased CKLF expression was also detected in CD4+ and CD8+ lymphocytes and was associated with $\mathrm{T}$ lymphocyte activation [23]. Data presented here confirms these findings, with detection of CKLF gene expression in CD4+ and CD8+ cells, but importantly indicates further increased expression within neutrophils, hematopoietic stem cells (HSCs) and a distinct class of T cells, namely the $\gamma \delta \mathrm{T}$ cell lineage. The role of neutrophils in CRC development has previously been shown to correlate with an adverse prognosis [24], but these findings have been challenged more recently, as neutrophils can be further subdivided into an anti-tumorigenic (N1) or pro-tumorigenic (N2) phenotype (reviewed in [25]). The CKLF expression level observed in HSCs, which is not maintained in all subpopulations of myeloid and lymphoid progenitor cells, warrants further investigation to determine the role that CKLF plays in this process of immune cell differentiation. Indeed, CKLF is increased following granulocyte colony-stimulating factor (G-CSF) stimulation, which causes mobilization of these cells into the bloodstream $[26,27]$. The $\gamma \delta$ T cell represents a unique immune population, which has been shown to play a key role in the immune system response to infection, cellular transformation and to tissue damage responses (reviewed in [28]). Although there is a paucity of data on the role of this cell lineage in mediating response to malignancy, [29] $\gamma \delta \mathrm{T}$ cells do exert a protective role in cancer. A limited number of studies have demonstrated the antitumorigenic role of $\gamma \delta$ T cells specifically in CRC $[30,31]$ with increased numbers of $\gamma \delta \mathrm{T}$ cells being recruited at the earliest stage of tumor initiation [32], while a further study has indicated their ability to protect the host from tumor formation in mouse models of malignancy [33].

While pathology-based studies have highlighted the favorable prognostic value associated with CD8+ T cell infiltration in CRC $[9,12]$, we now show for the first time that increased expression of CKLF has significant prognostic value in tumors classified as CMS1. It is possible that the increased levels of immune-derived CKLF are a surrogate marker for an increase in a specific type of immune cell infiltrate as suggested by our analyses. The specific anti-tumorigenic roles attributed to these particular classes of immune-infiltrate may also explain the poor prognosis observed in the CKLF-low subgroup of CMS1 tumors. While high levels of CKLF, indicating a high level of specific immune cell types, is sufficient to successfully eliminate these tumors, or at least hold them in a nascent equilibrium state, it is the lack of CKLF expression which suggests that without these immune cell populations within the TME, the neoplastic cells can escape the immune-surveillance and allow disease relapse [34]. These findings would require further detailed molecular pathology analysis in a large cohort of clinically annotated CMS1 tumor samples to validate. A limitation of hypothesis-driven studies such as this one is the requirement of open access to large stratified patient datasets, with matching molecular and clinical data, to allow hypothesis validation. While this issue will continue to limit the application of in silico clinical discovery strategies, the responsible but effective sharing of genomic and clinical data as espoused by, for example, the Global Alliance for Genomics and Health [35] to allow complete testing of this approach. Implementing such a molecular pathology "integromics" approach is becoming increasingly relevant in the era of stratified medicine [3638].

The importance of undertaking a stratification approach for molecular analyses, as described in this study, is further highlighted given the lack of prognostic value attributed to CKLF across the entire unstratified cohort. Without focused supervised analysis of patient transcriptional profiles, research approaches will continue to identify tumor factors which can identify poor prognostic tumor subgroups, such as the mesenchymal CMS4 tumors, based not on relapse risk but on overall tumor subtype. This approach inevitably leads to the assignment of patients into risk categories which are 
representative of the overall subgroup compared to the general population, but not based on the likelihood of individual relapse within the subgroup to which the tumor belongs, a finding which is far more clinically informative. This study also highlights the need for a radical shift in clinical trial design [21] coupled with a harmonized approach to biomarker development and patient stratification [39].

In conclusion, applying the recent consensus molecular subtypes to a large stage II/III patient cohort, we have extended the traditional pathology-based stratification of tumors into immune-high or immune-low tumors which have general prognostic value, with the identification of specific immune-derived factors, enabling us to understand the underlying biology accounting for these prognostic differences. Using this stratified diagnostics approach, we have identified CKLF as a favorable prognostic biomarker of relapse risk in the clinically relevant MSI-immune consensus molecular subtype of CRC. True validation of this type of hypothesis-driven biomarker discovery is reliant on further independent validation of these findings, in a molecular pathology-based analysis of prospective stratified clinical trial material.

\section{MATERIALS AND METHODS}

\section{Independent datasets}

Gene expression profiles from independent CRC datasets were downloaded from NCBI Gene Expression Omnibus (GEO) (http://www.ncbi.nlm.nih.gov/geo/) under accession numbers GSE39582, GSE14333, GSE39396 and GSE1133. GSE39582 contains 566 stage I-IV tumor profiles from a large CRC series, of which 460 stage II/III profiles are utilized in this study. GSE14333 contains 188 Dukes stage B/C profiles from mixed colon and rectal tumors. GSE39396 contains microarray profiles from fresh colorectal specimens where Fluorescence Activated Cell Sorting (FACS) selected cells into specific endothelial [CD45(+), EPCAM(-), CD31(-), FAP(-)], epithelial [CD45(-) $\operatorname{EPCAM}(+), \operatorname{CD} 31(-), \operatorname{FAP}(-)]$, leukocyte [CD45(-), $\operatorname{EPCAM}(-), \operatorname{CD} 31(+), \operatorname{FAP}(-)]$ and fibroblast $[\mathrm{CD} 45(-)$, EPCAM(-), CD31(-), FAP(+)] populations. GSE1133 consists of 79 human and 61 mouse tissue baseline gene expression microarray profiles. From this cohort, we selected the 79 human tissue transcriptional profiles. In addition to the NCBI cohorts, we have utilized both the GTEx and BioGPS portals. The RNA-seq data used for the analyses described in this manuscript were obtained from the Genotype-Tissue Expression (GTEx) portal (http://gtexportal.org/) version V6 in January 2016. This cohort consists of expression profiles from 53 human tissues across 8555 samples. The BioGPS database (http://biogps.org/) contains gene expression profiles across 745 samples representing a diverse range of primary human cells called the Expression Atlas of Human Primary Cells. This cohort was developed from combining a large number of publically available microarray datasets (745 samples, from over 100 separate studies) derived from human primary cells. Expression bar charts were plotted as median probeset values using GraphPad Prism version 5 for Windows.

\section{Risk assignment}

The study design and filters applied at each step are outlined in Figure 1. The entire GSE39582 cohort was filtered by excluding stage I and stage IV patient transcriptional profiles, followed by any transcriptional profiles with missing relapse data resulting in a cohort of 460 transcriptional profiles. For the discovery subset, we removed patient transcriptional profiles which were censored to follow up prior to 36 months (unknown relapse data). Patients that relapsed prior to 36 months were classified as high-risk and patients with no relapse were classified as low risk, resulting in 372 transcriptional profiles. This discovery subset was further filtered to contain only transcriptional profiles from untreated patients, resulting in 177 transcriptional profiles. Three year relapse free survival analysis was performed on these 177 transcriptional profiles to determine relapse rate information across all CMS assigned tumors using GraphPad Prism version 5 for Windows. This 177 transcriptional profile subgroup was then further filtered to contain only CMS1 assigned transcriptional profiles resulting in 46 tumor transcriptional profiles, 6 assigned as high risk and 40 with low risk assignment (Figure 1).

\section{Transcriptional analysis}

Partek Genomics Suite was used for dataset analysis. Differentially expressed probesets which had a fold-change $+/-1.5$ fold and $p$-value $<0.005$ were defined using analysis of variance (ANOVA) of supervised risk groupings. For the purpose of clustering, the data matrices were standardized to the median value of probeset expression. Standardization of the data allows for comparison of expression levels for different probesets, which may not necessarily be on the same scale or at the same intensity levels. Following standardization, 2-dimensional hierarchical clustering was performed (samples $\mathrm{x}$ probe sets/genes). Euclidean distance was used to calculate the distance matrix, which is a multidimensional matrix representing the distance from each data point (probe set-sample pair) to all the other data points. Ward's linkage method was subsequently applied to join the samples and genes together, with the minimum variance, to find compact clusters based on the calculated distance matrix. 


\section{Chemokine-like factor (CKLF) stratification}

Tertile stratification was performed on the mean CKLF expression value from the three probesets used within the CMS1, MSI and complete cohorts. These values were classified as high, medium and low based on 1:1:1 sample distributions. In the case where there were uneven numbers for equal distribution, preference was given to equally distribute the high and low groups.

\section{Survival analysis}

Survival validation was performed on the CMS1 $(\mathrm{n}=79)$ and MSI $(\mathrm{n}=60)$ transcriptional profiles from the 460 stage II/III transcriptional profiles with complete clinical information. Survival curves, comparing expression and treatment subgroups were plotted from patient details right censored at 36 months, to give 3-year relapse rates using GraphPad Prism version 5 for Windows. Cox Proportional Hazards analysis, using Stata version 11.2, was applied to evaluate recurrence-free survival according to CKLF gene expression levels within the CMS1 subgroup, within MSI tumors and in the entire cohort, prior to and after adjustment for age, sex, tumor stage and location, and receipt of adjuvant treatment. Stratified analysis was also conducted in untreated patients only, using the relapse data for the duration of follow up within the dataset (201 months maximum follow up). Categorical and continuous variables were compared between individuals with CMS1 tumors and the rest of the overall cohort using chi-squared tests and t-tests, respectively.

\section{CONFLICTS OF INTEREST}

PDD: None; POR: None; HGC: None; RTG: None; DBL: None; PGJ: Previous Founder and Shareholder of Almac Diagnostics; CV6 Therapeutics: Expert Advisor and Shareholder; Chugai Pharmaceuticals: Consultant; MST: None; ML: None; DMA: None.

\section{FINANCIAL SUPPORT}

This work was supported by a Cancer Research UK (CRUK) Programme Grant and a joint Medical Research Council (MRC) / CRUK stratified medicine grant (S:CORT).

\section{REFERENCES}

1. Perou CM, Sorlie T, Eisen MB, van de Rijn M, Jeffrey SS, Rees CA, Pollack JR, Ross DT, Johnsen H, Akslen LA, Fluge $\mathrm{O}$, Pergamenschikov A, Williams C, et al. Molecular portraits of human breast tumours. Nature. 2000; 406: 747-752.

2. Sorlie T, Perou CM, Tibshirani R, Aas T, Geisler S, Johnsen H, Hastie T, Eisen MB, van de Rijn M, Jeffrey SS, Thorsen T, Quist H, Matese JC, et al. Gene expression patterns of breast carcinomas distinguish tumor subclasses with clinical implications. Proceedings of the National Academy of Sciences of the United States of America. 2001; 98: 10869-10874.

3. Cancer Genome Atlas Network. Comprehensive molecular characterization of human colon and rectal cancer. Nature. 2012; 487: 330-337.

4. Roepman P, Schlicker A, Tabernero J, Majewski I, Tian S, Moreno V, Snel MH, Chresta CM, Rosenberg R, Nitsche U, Macarulla T, Capella G, Salazar R, et al. Colorectal cancer intrinsic subtypes predict chemotherapy benefit, deficient mismatch repair and epithelial-to-mesenchymal transition. International journal of cancer. 2014; 134: 552-562.

5. Sadanandam A, Lyssiotis CA, Homicsko K, Collisson EA, Gibb WJ, Wullschleger S, Ostos LC, Lannon WA, Grotzinger C, Del Rio M, Lhermitte B, Olshen AB, Wiedenmann B, et al. A colorectal cancer classification system that associates cellular phenotype and responses to therapy. Nature medicine. 2013; 19: 619-625.

6. de Sousa E Melo F, Colak S, Buikhuisen J, Koster J, Cameron K, de Jong JH, Tuynman JB, Prasetyanti PR, Fessler E, van den Bergh SP, Rodermond H, Dekker E, van der Loos CM, et al. Methylation of cancer-stemcell-associated Wnt target genes predicts poor prognosis in colorectal cancer patients. Cell stem cell. 2011; 9: 476-485.

7. Marisa L, de Reynies A, Duval A, Selves J, Gaub MP, Vescovo L, Etienne-Grimaldi MC, Schiappa R, Guenot D, Ayadi M, Kirzin S, Chazal M, Flejou JF, et al. Gene expression classification of colon cancer into molecular subtypes: characterization, validation, and prognostic value. PLoS medicine. 2013; 10: e1001453.

8. Guinney J, Dienstmann R, Wang X, de Reynies A, Schlicker A, Soneson C, Marisa L, Roepman P, Nyamundanda G, Angelino P, Bot BM, Morris JS, Simon IM, et al. The consensus molecular subtypes of colorectal cancer. Nature medicine. 2015.

9. Galon J, Costes A, Sanchez-Cabo F, Kirilovsky A, Mlecnik B, Lagorce-Pages C, Tosolini M, Camus M, Berger A, Wind P, Zinzindohoue F, Bruneval P, Cugnenc PH, et al. Type, density, and location of immune cells within human colorectal tumors predict clinical outcome. Science (New York, N.Y.). 2006; 313: 1960-1964.

10. Thibodeau SN, Bren G, Schaid D. Microsatellite instability in cancer of the proximal colon. Science (New York, N.Y.). 1993; 260: 816-819.

11. Boland CR, Goel A. Microsatellite instability in colorectal cancer. Gastroenterology. 2010; 138: 2073-2087.e3.

12. Pages F, Berger A, Camus M, Sanchez-Cabo F, Costes A, Molidor R, Mlecnik B, Kirilovsky A, Nilsson M, Damotte D, Meatchi T, Bruneval P, Cugnenc PH, et al. Effector memory $\mathrm{T}$ cells, early metastasis, and survival in colorectal cancer. The New England journal of medicine. 2005; 353: 2654-2666.

13. Gentles AJ, Newman AM, Liu CL, Bratman SV, Feng W, Kim D, Nair VS, Xu Y, Khuong A, Hoang CD, Diehn M, 
West RB, Plevritis SK, et al. The prognostic landscape of genes and infiltrating immune cells across human cancers. Nature medicine. 2015; 21: 938-945.

14. Fridman WH, Pages F, Sautes-Fridman C, Galon J. The immune contexture in human tumours: impact on clinical outcome. Nature reviews. Cancer. 2012; 12: 298-306.

15. Calon A, Espinet E, Palomo-Ponce S, Tauriello DV, Iglesias M, Cespedes MV, Sevillano M, Nadal C, Jung P, Zhang XH, Byrom D, Riera A, Rossell D, et al. Dependency of colorectal cancer on a TGF-beta-driven program in stromal cells for metastasis initiation. Cancer cell. 2012; 22: 571-584.

16. GTEx Consortium. Human genomics. The GenotypeTissue Expression (GTEx) pilot analysis: multitissue gene regulation in humans. Science (New York, N.Y.). 2015; 348: 648-660.

17. Mele M, Ferreira PG, Reverter F, DeLuca DS, Monlong J, Sammeth M, Young TR, Goldmann JM, Pervouchine DD, Sullivan TJ, Johnson R, Segre AV, Djebali S, et al. Human genomics. The human transcriptome across tissues and individuals. Science (New York, N.Y.). 2015; 348: 660-665.

18. Su AI, Wiltshire T, Batalov S, Lapp H, Ching KA, Block D, Zhang J, Soden R, Hayakawa M, Kreiman G, Cooke MP, Walker JR, Hogenesch JB. A gene atlas of the mouse and human protein-encoding transcriptomes. Proceedings of the National Academy of Sciences of the United States of America. 2004; 101: 6062-6067.

19. Wu C, Orozco C, Boyer J, Leglise M, Goodale J, Batalov S, Hodge CL, Haase J, Janes J, Huss JW,3rd, Su AI. BioGPS: an extensible and customizable portal for querying and organizing gene annotation resources. Genome biology. 2009; 10: R130-2009-10-11-r130. Epub 2009 Nov 17.

20. Mabbott NA, Baillie JK, Brown H, Freeman TC, Hume DA. An expression atlas of human primary cells: inference of gene function from coexpression networks. BMC genomics. 2013; 14: 632-2164-14-632.

21. Lawler M, Kaplan R, Wilson RH, Maughan T, S-CORT Consortium. Changing the Paradigm-Multistage Multiarm Randomized Trials and Stratified Cancer Medicine. The oncologist. 2015; 20: 849-851.

22. Han W, Lou Y, Tang J, Zhang Y, Chen Y, Li Y, Gu W, Huang J, Gui L, Tang Y, Li F, Song Q, Di C, et al. Molecular cloning and characterization of chemokinelike factor 1 (CKLF1), a novel human cytokine with unique structure and potential chemotactic activity. The Biochemical journal. 2001; 357: 127-135.

23. Li T, Zhong J, Chen Y, Qiu X, Zhang T, Ma D, Han W. Expression of chemokine-like factor 1 is upregulated during T lymphocyte activation. Life Sciences. 2006; 79: 519-524.

24. Rao HL, Chen JW, Li M, Xiao YB, Fu J, Zeng YX, Cai MY, Xie D. Increased intratumoral neutrophil in colorectal carcinomas correlates closely with malignant phenotype and predicts patients' adverse prognosis. PloS one. 2012; 7: e30806.

25. Fridlender ZG, Albelda SM. Tumor-associated neutrophils: friend or foe?. Carcinogenesis. 2012; 33: 949-955.
26. Levesque JP, Winkler IG, Larsen SR, Rasko JE. Mobilization of bone marrow-derived progenitors. Handbook of Experimental Pharmacology. 2007; (180): 3-36.

27. Thomas J, Liu F, Link DC. Mechanisms of mobilization of hematopoietic progenitors with granulocyte colony-stimulating factor. Current opinion in hematology. 2002; 9: 183-189.

28. Vantourout P, Hayday A. Six-of-the-best: unique contributions of gammadelta $\mathrm{T}$ cells to immunology. Nature reviews.Immunology. 2013; 13: 88-100.

29. Silva-Santos B, Serre K, Norell H. gammadelta T cells in cancer. Nature reviews. Immunology. 2015; 15: 683-691.

30. Corvaisier M, Moreau-Aubry A, Diez E, Bennouna J, Mosnier JF, Scotet E, Bonneville M, Jotereau F. V gamma $9 \mathrm{~V}$ delta $2 \mathrm{~T}$ cell response to colon carcinoma cells. Journal of immunology (Baltimore, Md.: 1950). 2005; 175: 5481-5488.

31. Todaro M, D'Asaro M, Caccamo N, Iovino F, Francipane MG, Meraviglia S, Orlando V, La Mendola C, Gulotta G, Salerno A, Dieli F, Stassi G. Efficient killing of human colon cancer stem cells by gammadelta T lymphocytes. Journal of immunology (Baltimore, Md.: 1950). 2009; 182: 7287-7296.

32. Gao Y, Yang W, Pan M, Scully E, Girardi M, Augenlicht LH, Craft J, Yin Z. Gamma delta T cells provide an early source of interferon gamma in tumor immunity. The Journal of experimental medicine. 2003; 198: 433-442.

33. Girardi M, Glusac E, Filler RB, Roberts SJ, Propperova I, Lewis J, Tigelaar RE, Hayday AC. The distinct contributions of murine $\mathrm{T}$ cell receptor (TCR)gammadelta+ and TCRalphabeta+ $\mathrm{T}$ cells to different stages of chemically induced skin cancer. The Journal of experimental medicine. 2003; 198: 747-755.

34. Dunn GP, Bruce AT, Ikeda H, Old LJ, Schreiber RD. Cancer immunoediting: from immunosurveillance to tumor escape. Nature immunology. 2002; 3: 991-998.

35. Lawler M, Siu LL, Rehm HL, Chanock SJ, Alterovitz G, Burn J, Calvo F, Lacombe D, Teh BT, North KN, Sawyers CL, Clinical Working Group of the Global Alliance for Genomics and Health (GA4GH). All the World's a Stage: Facilitating Discovery Science and Improved Cancer Care through the Global Alliance for Genomics and Health. Cancer discovery. 2015; 5: 1133-1136.

36. Salto-Tellez M, Kennedy RD. Integrated molecular pathology: the Belfast model. Drug discovery today. 2015.

37. Salto-Tellez M, James JA, Hamilton PW. Molecular pathology - the value of an integrative approach. Molecular oncology. 2014; 8: 1163-1168.

38. McArt DG, Blayney JK, Boyle DP, Irwin GW, Moran M, Hutchinson RA, Bankhead P, Kieran D, Wang Y, Dunne PD, Kennedy RD, Mullan PB, Harkin DP, et al. PICan: An integromics framework for dynamic cancer biomarker discovery. Molecular oncology. 2015; 9: 1234-1240.

39. Schneider D, Bianchini G, Horgan D, Michiels S, Witjes W, Hills R, Plun-Favreau J, Brand A, Lawler M, EAPM Working Group for Oncology Clinical Research. Establishing the Evidence Bar for Molecular Diagnostics in Personalised Cancer Care. Public health genomics. 2015; 18: 349-358. 